\title{
A NEW DUALITY THEOREM FOR SEMISIMPLE MODULES AND CHARACTERIZATION OF VILLAMAYOR RINGS
}

\author{
CARL FAITH AND PERE MENAL
}

(Communicated by Lance W. Small)

\begin{abstract}
We prove the theorem: If $R$ is a ring whose right ideals satisfy the double annihilator condition with respect to a semisimple right $R$-module $W$, then every right ideal is an intersection of maximal right ideals, consequently $R$ is a right $V$ (for Villamayor) ring, and $W$ is then necessarily a cogenerator of mod- $R$. (The converse is well known.) We use this to give a new proof of a theorem of ours on right Johns rings.
\end{abstract}

\section{INTRODUCTION}

A ring $R$ is a right $V$ ring provided that $R$ satisfies the f.e.c.'s:

(V1) Every simple right $R$-module is injective.

(V2) Every right ideal $I$ of $R$ is an intersection of maximal right ideals, equivalently, $R / I$ has zero Jacobson radical, i.e., $\operatorname{rad}(R / I)=0$.

(V3) Every right $R$-module $M$ has zero Jacobson radical, i.e., $\operatorname{rad} M=0$.

Proof. See [F1], p. 356, Proposition 7.32A.

If $W$ is a right $R$-module, we say that $W$ satisfies the double annihilator condition $(=$ d.a.c. $)$ with respect to right ideals provided that

$$
I=\operatorname{ann}_{R} \operatorname{ann}_{W} I
$$

where $\operatorname{ann}_{W} S$ denotes the annihilator of a subset $S$ of $R$ in $W$, and dually for $\operatorname{ann}_{R} S$ for a subset $S$ of $W$.

\section{A CHARACTERIZATION OF $V$-RINGS}

We now state the new characterization of $V$-rings.

$V$-Ring Theorem. $A$ ring $R$ is a right $V$-ring iff some semisimple right $R$ module satisfies the d.a.c. with respect to right ideals.

Proof. Sufficiency. It suffices to prove that every right ideal of $I$ of $R$ is the intersection of maximal right ideals. Let $M=\mathrm{ann}_{W} I$. Then by the d.a.c.

$$
I=\bigcap_{m \in M} \operatorname{ann}_{R} m
$$

Received by the editors September 21, 1993.

1991 Mathematics Subject Classification. Primary 16D60, 16D10; Secondary 16D50, 16G99.

The second author is deceased (April 4, 1991; see notes in [F-M1] and [P]). 
Since $m R$ is a semisimple $R$-submodule of finite length, then

$$
m R=v_{1} R \oplus \cdots \oplus v_{n} R
$$

where $v_{i} \in W$ and $v_{i} R$ is simple, $i=1, \ldots, n$. Write

$$
m=v_{1} a_{1}+\cdots+v_{n} a_{n}
$$

where $a_{i} \in R, i=1, \ldots, n$; and let

$$
H=\bigcap_{i=1}^{n} \operatorname{ann}_{R} v_{i} a_{i} .
$$

We assert that

$$
H=\operatorname{ann}_{R} m
$$

is the intersection of maximal right ideals. For if $v_{i} a_{i} \neq 0$, then $v_{i} a_{i} R=v_{i} R$ is simple, and since

$$
v_{i} R \approx R /\left(\operatorname{ann}_{R} v_{i} a_{i}\right),
$$

then $\operatorname{ann}_{R} v_{i} a_{i}$ is a maximal right ideal, hence $H$ is the intersection of maximal right ideals.

Next we show that (5) holds. Obviously, $\operatorname{ann}_{R} m \supseteq H$. To prove the reverse inclusion, note that if $r \in \operatorname{ann}_{R} m$, then

$$
v_{1} a_{1} r+\cdots+v_{n} a_{n} r=0 .
$$

By (2), then $v_{i} a_{i} r=0$, hence $r \in \operatorname{ann}_{R} v_{i} a_{i}$ for all $i$, that is, $r \in H$, so (5) holds.

Since (4) is the intersection of maximal right ideals, then so is $\operatorname{ann}_{R} m$, whence $I$ by (1).

Necessity. If $R$ is a right $V$-ring, then the direct sum $W$ of a complete isomorphic set of simple right $R$-modules is a minimal cogenerator of mod-R . (See, e.g., [F1], p. 167, Proposition 3.55. There is a misprint in this proposition; cf. [F2].) Furthermore, every cogenerator $W$ satisfies the d.a.c. with respect to right ideals. (Hint: if $I$ is a right ideal, then $R / I$ embeds in a direct product $W^{\alpha}$ of copies of $W$, say $h: R / I \mapsto W^{\alpha}$. If $h(1+I)=\left(w_{i}\right) \in W^{\alpha}$, then $\left.I=\operatorname{ann}_{R}\left\{w_{i}\right\}_{i \in \alpha}.\right)$

Corollary. If a ring $R$ satisfies the d.a.c. with respect to a semisimple right $R$ module $W$, then $W$ is a right cogenerator of $R$.

Proof. $R$ is a right $V$-ring, so every simple right $R$-module $V$ is injective, so it suffices to show that $W$ contains a copy of each such $V$. But $V \approx R / M$, where $M \triangleleft R$, and by the d.a.c., $M=\operatorname{ann}_{R} w$ for some $w \in W$. Since $w R \approx V$, we have $V \hookrightarrow W$ as needed.

F-M Theorem 2.3 ([F-M1]). If $R$ is a right Johns ring (= right Noetherian and every right ideal is a right annihilator), then $R / J$ is a right $V$-ring, where $J$ is the Jacobson radical.

Proof. Let $W=\operatorname{soc} R$. Then

$$
J={ }^{\perp} W=W^{\perp}
$$

is nilpotent and

$$
W=J^{\perp}={ }^{\perp} J
$$


where " $\perp$ " denotes an annihilator in $R$ on the appropriate side. (See [F-M], Lemma 2.2.)

By the fact that $R_{R}$ satisfies the d.a.c. with respect to right ideals, and using (*) and (**), one sees that right ideals of $R$ containing $J$, hence right ideals of $R / J$, satisfy the d.a.c. with respect to the semisimple module $W$. Then by the $V$-Ring Theorem, we see that $R / J$ is a right $V$-ring.

Corollary. If $R$ is such that $W={ }^{\perp} J$ is a semisimple right $R$-module and every right ideal containing $J$ is a right annihilator, then $R / J$ is a right $V$-ring.

\section{ACKNOWLEDGMENT}

We wish to acknowledge P. M. Cohn for raising a question about the proof of Theorem 2.3, in connection with his reviewing [F-M1], namely, given a finitely embedded (= f.e.) module $M$ (= has finite essential socle) that occurs in the proof, and given an embedding of $M$ in a direct product $S^{\alpha}$ of copies of a module ( $S=\operatorname{soc} R_{R}$ in the proof), how does one conclude that $M$ embeds in a finite product $S^{n}$ of copies of $S$ ? This can be resolved as follows. Let $\left\{p_{i}\right\}_{i \in \alpha}$ denote the set of projections $S^{\alpha} \rightarrow S$ of the product $S^{\alpha}$. Since $\bigcap_{i \in \alpha} \operatorname{ker} p_{i}=$ 0 , and since $M$ is f.e., then for some finite subset $\bar{p}_{i_{1}}, \ldots, \bar{p}_{i_{n}}$ of the induced maps $\bar{p}_{i}: M \rightarrow S$ we have $\bigcap_{i=1}^{n}$ ker $\bar{p}_{i}=0$. But then the direct sum of the $\left\{\bar{p}_{i}\right\}_{i=1}^{n}$ is an embedding $M \rightarrow S^{n}$.

\section{REFERENCES}

[F1] C. Faith, Algebra I: Rings, modules and categories, Grundlehren Math. Wiss., Bd. 190, Springer-Verlag, Berlin, Heidelberg, and New York, 1973 (corrected reprint, 1981).

[F2] - Minimal cogenerators over Osofsky and Camillo rings, preprint, 1993.

[F-M1] C. Faith and Pere Menal, A counter-example to a conjecture of Johns, Proc. Amer. Math. Soc. 116 (1992), 21-26.

[F-M2] _ The structure of Johns rings, Proc. Amer. Math. Soc. 120 (1994), 1071-1081.

[P] C. Perelló (ed.), Pere Menal memorial volumes, Publ. Mat. 36 (1992).

Department of Mathematics, Rutgers University New Brunswick, New Jersey 08903

Permanent address: 199 Longview Drive, Princeton, New Jersey 08540 